\title{
Invasive meningococcal disease in Malta: an epidemiological overview, 1994-2007
}

\author{
Mark Muscat, ${ }^{1}$ Gianfranco Spiteri, ${ }^{2}$ Neville Calleja, ${ }^{3}$ Julie Haider, ${ }^{4}$ \\ Stephen J. Gray, ${ }^{5}$ Jackie Maistre Melillo, ${ }^{2}$ Julian Mamo ${ }^{6}$ \\ and Paul Cuschieri ${ }^{4}$
}

Correspondence

Mark Muscat mmc@ssi.dk

Received 15 April 2009 Accepted 9 July 2009

\begin{abstract}
${ }^{1}$ Department of Epidemiology, Statens Serum Institut, Artillerivej 5, DK-2300 Copenhagen S, Denmark

${ }^{2}$ Infectious Disease Prevention and Control Unit, Department of Health Promotion and Disease Prevention, 5B The Emporium, C. de Brocktorff Street, Msida MSD 1421, Malta

${ }^{3}$ Directorate of Health Information and Research, 95 G'Mangia Hill, G'Mangia PTA 1313, Malta

${ }^{4}$ Bacteriology Laboratory, Department of Pathology, Mater Dei Hospital, Msida MSD 2090, Malta

${ }^{5}$ Meningococcal Reference Unit, Health Protection Agency, Manchester Medical Microbiology Partnership, Manchester Royal Infirmary, Oxford Road, Manchester M13 9WZ, UK

${ }^{6}$ Department of Public Health, Mater Dei Hospital, Medical School, University of Malta, Msida MSD 2090, Malta
\end{abstract}

\begin{abstract}
Since 1996, Malta has experienced an upsurge of invasive meningococcal disease (IMD) following an almost 30 year period with a negligible number of annually reported cases. We reviewed the 233 IMD cases notified during a 14 year period (1994-2007), and analysed epidemiological and laboratory surveillance data. The crude incidence per 100000 inhabitants peaked in 2000 at 8.1 [95\% confidence interval $(\mathrm{Cl}) 5.7-11.6$ ] and again in 2006 at $8.9(95 \% \mathrm{Cl}$ 6.4-12.4), thereby placing Malta amongst the countries with the highest incidence of the disease in Europe. Of the total cases, 137 (59\%) were confirmed and 30 (13\%) were classified as probable. However, 66 cases $(28 \%)$ had no laboratory evidence of the disease and were classified as possible. Information on the serogroup was available for 114 cases. Serogroup $B$ formed the largest proportion (76\%,n=87) followed by serogroup $\mathrm{C}(16 \%, n=18)$.

$\mathrm{B}: 4: \mathrm{P} 1.19,15$ strains $(n=46)$ predominated throughout the study period since their first identification in 1998. With 28 deaths attributed to IMD, the overall case fatality rate was $12 \%$. Apart from stressing the importance of maintaining high vigilance for IMD, our findings underscore the importance of enhancing laboratory surveillance of the disease, including characterization of the meningococci. Until vaccines against a broad range of serogroup $B$ meningococci become available for universal use, the main methods of control remain the early treatment of cases and the prevention of secondary cases.
\end{abstract}

\section{INTRODUCTION}

The Mediterranean island country of Malta has experienced an upsurge of invasive meningococcal disease (IMD) following an almost 30 year period with zero to three cases reported annually (COS, 1971-1986; NSO, 1987-1994). Before this time, the highest incidence of the disease was last recorded during the Second World War when it was notified as cerebrospinal fever (DH, 1933-1970). Apart

Abbreviations: CDC, Centers for Disease Control and Prevention; CFR, case fatality rate; $\mathrm{Cl}$, confidence interval; CSF, cerebrospinal fluid; IDCU, Infectious Disease Prevention and Control Unit; IMD, invasive meningococcal disease; OMP, outer-membrane protein. from a historical account of the disease's first appearance in Malta in the late 19th century (Savona-Ventura, 1994), scientific literature on the disease in Malta is scarce. The purpose of this study was to describe the epidemiology of IMD in Malta in the 14 year period 1994-2007, during which the incidence of the disease rose to one of the highest incidence levels in Europe (EU-IBIS, 2007).

With a total area of $316 \mathrm{~km}^{2}$ and an estimated total population of just over 400000 [Demographic Review 2007, National Statistics Office, Malta, 2008 (http://www. nso.gov.mt/statdoc/document_file.aspx?id=2325 - accessed 8 September 2008)], Malta has one of the highest population densities in Europe $\left(>1200\right.$ persons $\left.\mathrm{km}^{-2}\right)$. 
Moreover, the country is visited by over one million tourists annually [Tourism 2001 - an Overview, National Statistics Office, Malta, 2002 (http://www.nso.gov.mt/ statdoc/document_file.aspx?id=740 - accessed 18 July 2008)]. Malta is a developed nation with health indicators on a par with other European countries and good standards of public hygiene [European Health for All Database, World Health Organization Regional Office for Europe, Copenhagen (http://www.euro.who.int/hfadb accessed 22 May 2009)].

IMD is the result of a systemic bacterial infection by the Gram-negative diplococcus Neisseria meningitidis, commonly referred to as meningococcus. Meningococci are classified into a number of serogroups based on structural differences in the capsular polysaccharides with serogroups $\mathrm{B}$ and $\mathrm{C}$ responsible for the majority of cases in Europe (Stephens et al., 2007).

Further phenotypic characterization of meningococci includes that made on the basis of their structural class 2 and 3 outer-membrane proteins (OMPs) designated PorB, which defines serotypes, and class 1 OMP (PorA), which determines sero-subtypes. The PorA phenotype (class 1 OMP) may be described by reactivity to specific mAbs at three variable regions (VR1, VR2 and VR3), but the limited panel of reagents available often only detects the VR1 and VR2 peptide sequences or epitopes (Gray et al., 2006). The designation of a particular phenotype follows the order of serogroup, serotype and sero-subtypes, for example B : $4:$ P1.19,15 - with two separate sero-subtyping epitopes P1.19 and P1.15 for VR1 and VR2, respectively.

PCR analysis has also proven to be useful for both the laboratory confirmation and characterization of meningococcal phenotypes (Diggle \& Clarke, 2006). However, recent studies have shown the benefits of increased discrimination using DNA sequence-based approaches such as multilocus sequence typing (Russell et al., 2008). The distribution of the various strains of meningococci differs with age, time and geographical location (Tikhomirov et al., 1997; Van Deuren et al., 2000).

Clinically, IMD most commonly presents as meningitis or septicaemia, or a combination of both. Other less common presentations of meningococcal disease include pneumonia, myocarditis, endocarditis, pericarditis, arthritis, conjunctivitis, urethritis, pharyngitis and cervicitis (Rosenstein et al., 2001). The high case fatality rate (CFR) of IMD (Sharip et al., 2006) continues to cause great public concern and attracts considerable media attention.

Although a vaccine against serogroup $\mathrm{C}$ meningococcal disease has been developed and is used in several European countries (EU-IBIS, 2007) there is, as yet, no effective vaccine currently available for universal use against a broad range of serogroup B meningococci (Stephens et al., 2007). In Malta, the only preventive measure taken against the disease is the targeting of the contacts of the patients with chemoprophylaxis, and where relevant, with polysaccharide meningococcal vaccines available for the prevention of serogroup A, C, Y and $\mathrm{W}-135$ disease.

\section{METHODS}

Study population. We included Maltese residents notified as having IMD during January 1994 through December 2007. A confirmed case of IMD was defined as a notified case with a clinical diagnosis of meningitis and/or septicaemia, with the isolation of $N$. meningitidis or the detection of Gram-negative diplococci from a sterile site such as blood, cerebrospinal fluid (CSF) or joint fluid. Inclusion as a probable case required a clinical diagnosis of meningitis and/or septicaemia, with the isolation of $N$. meningitidis or the detection of Gramnegative diplococci from a non-sterile site (such as skin scrapings of purpuric lesions and throat swab), or a positive rapid antigen screen (agglutination test) for N. meningitidis on CSF. Notified IMD cases without any laboratory evidence for confirmation were considered as possible cases. Non-residents and cases of patients with IMD other than meningitis and/or septicaemia were excluded.

Data collection. IMD is a statutory notifiable disease in Malta. We obtained data on IMD from the Infectious Disease Prevention and Control Unit (IDCU), which is the national competent body for surveillance of infectious diseases in Malta. The IDCU carries out routine passive surveillance based on notifications received from medical practitioners and laboratories. Deaths attributed to IMD were notified to the IDCU directly by hospital staff and officially through the Directorate of Health Information and Research, which processes all death certificates. In Malta, the certification of deaths and cause thereof is the legal duty of medical practitioners [Malta Government Legal Notice 11, Certificate of Death and Cause of Death Regulations, 2008; 465 of the Public Health Act, 2008, Government Gazette of Malta 18 175. http://doi.gov.mt/en/legalnotices/2008/01/LN\%2011. pdf (accessed on 25 May 2009)].

For each notified case the IDCU collates epidemiological, clinical, laboratory and mortality data. Since management, laboratory investigation and notification of IMD cases in Malta is centralized at national level, reporting of cases to the IDCU is assumed to be complete. No changes in the epidemiological surveillance practice for IMD have occurred during the study period. The study was approved by the Health Ethics Committee of Malta.

Characterization of meningococci. Serogrouping was carried out using slide agglutination tests based on serogroup-specific antisera (Meningococcus agglutinating sera; Remel Europe) on culture isolates or by using commercial latex agglutination tests (Pastorex Meningitis 25 test kit; Bio-Rad Laboratories; or Wellcogen bacterial antigen kit; Murex Biotech) on CSF supernatant where available. Since 1998, isolates from the majority of culture-positive cases $(87 / 102 ; 85 \%)$ were submitted to the Meningococcal Reference Unit of the Health Protection Agency, UK, for phenotyping and antibiotic-susceptibility testing. The serotype mAbs used included: $1,2 \mathrm{a}, 2 \mathrm{~b}, 4 \mathrm{p}, 4 \mathrm{z}, 11,14,15$, 21 and 22 (Gray et al., 2006). The sero-subtype mAbs used included: P1.1, P1.2, P1.3, P1.4, P1.5, P1.6, P1.7, P1.9, P1.10, P1.12, P1.13, P1.14, P1.15, P1.16 and P1.19 (Gray et al., 2006). The results of the antibiotic-susceptibility testing are not presented.

Disease classification. Based primarily on the presenting clinical features, we classified IMD cases as meningitis, septicaemia, or a mixed picture of both. Cases of meningitis with a positive culture from blood samples were classified in the latter group.

Statistical methods. Incidence was calculated with the number of cases of IMD as numerator and population data, obtained from the 
National Statistics Office of Malta, as the denominator. To assess statistical significance of time trends in age-specific incidence, we used Poisson regression. Independence can be assumed from the data because, with the exception of two epidemiologically linked cases, and one case of a patient who suffered IMD twice, all were sporadic throughout the study period. Analyses were performed with the cases grouped into 2 year intervals to diminish any existing correlation between sequential years. The association between serogroup and disease classification, and serogroup and mortality was evaluated by chi-square testing using Epi Info 2002 software. Confidence intervals (CIs) (at 95\%) were calculated around the estimated rates. To examine for seasonality we analysed cases by disease onset in quarterly periods for all notified cases, and on those with a confirmed and probable status, using non-parametric Kruskal-Wallis and median tests with SPSS software (version 16).

\section{RESULTS AND DISCUSSION}

During 1994-2007, a total of 233 IMD cases was notified. Of these, 137 (59\%) were confirmed, 30 (13\%) were probable and the remaining $66(28 \%)$ were classified as possible cases. The crude incidence of notified IMD per 100000 inhabitants peaked in 2000 at 8.1 (95\% CI 5.7$11.6)$ and again in 2006 at 8.9 (95\% CI 6.4-12.4) (Fig. 1). The upsurge of IMD became noticeable in 1996, placing Malta with Ireland and Iceland as countries with markedly higher incidence rates than other European countries during the same period (Trotter et al., 2007). Given the small population of Malta, minor changes in the number of cases cause marked swings in incidence. Nevertheless, with persistently high IMD incidence rates, Malta became hyper-endemic for the disease. There is no single clear explanation for this resurgence. Studies have clearly shown increased incidence rates following the introduction of new strains of N. meningitidis into the populations of Canada (Ashton et al., 1991), the Czech Republic (Krïzová et al., 1997), Denmark (Lind \& Berthelsen, 2005), Greece (Kremastinou et al., 1999), Norway (Poolman et al., 1986) and Spain (Berron et al., 1998).

With the exception of an epidemiological link between two cases of patients from the same household, the cases were

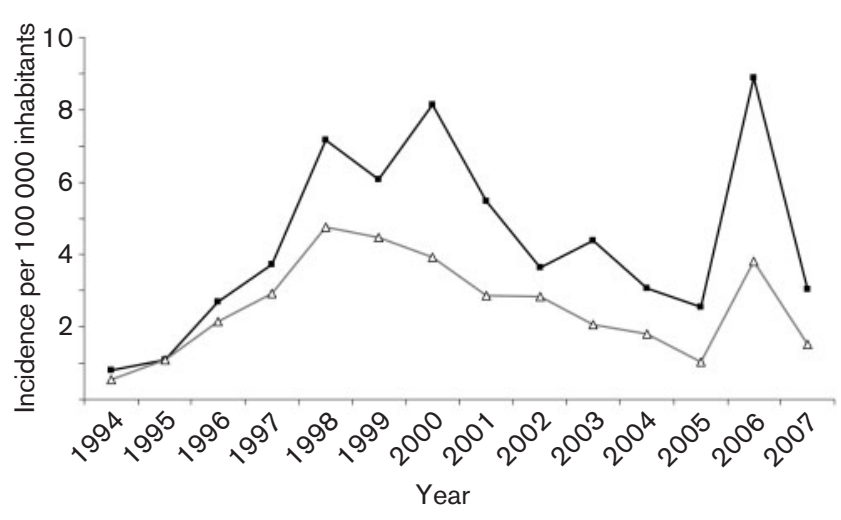

Fig. 1. Incidence of cases of notified $(n=233)$ and confirmed ( $n=137)$ IMD, 1994-2007. $\square$, Notified cases; $\triangle$, confirmed cases. considered as sporadic. One case, of a patient later diagnosed with complement deficiency, was reported with IMD in 1995 and again in 1999. In another two cases, both involving children, a diagnosis of rickettsiosis had originally been made only until meningococci were isolated from blood cultures. Both patients had responded successfully to ciprofloxacin with which they were treated in favour of a tetracycline, which is contraindicated in young children.

Of the confirmed cases, 105 (77\%) were culture positive from a normally sterile site. Of the probable cases, 13 $(43 \%)$ had a positive culture from a non-sterile site: 11 from throat swabs and 2 from skin lesion material. Information on the serogroup was available for 114 cases of which $87(76.3 \%)$ were serogroup B, $18(15.8 \%)$ were serogroup C, $4(3.5 \%)$ were serogroup $\mathrm{W}-135$ and 1 $(0.9 \%)$ was serogroup D. In four cases $(3.5 \%)$ the isolates were non-groupable (Fig. 2). One case originally classified as serogroup A gave a weak auto-agglutination reaction; however, upon further testing at the reference laboratory it was confirmed as a non-groupable case.

Tables 1 and 2 show the annual distribution of serogroup B and $\mathrm{C}$ cases by serotype and sero-subtype. Of the serogroup B isolates, for $72(83 \%)$ there was information on serotype, of which $52(72 \%)$ were serotype 4 . Of these, 49 had a known sero-subtype for both VR1 and VR2 epitopes and included 46 (94\%) B:4:P1.19,15 strains. The rest $(6 \%)$ were $\mathrm{B}: 4: \mathrm{P} 1.12,15, \mathrm{~B}: 4: \mathrm{P} 1.19,13$ and $\mathrm{B}: 4$ : NT,P1.13 strains.

Serogroup B was the predominant strain as in the rest of Europe during the same period (Connolly \& Noah, 1999; Trotter et al., 2007). Our findings show that B:4 strains with the combined P1.19 and P1.15 sero-subtype epitopes have dominated since their first identification in Malta in 1998. The presence of $\mathrm{B}: 4: \mathrm{P} 1.19,15$ strains has also been reported in Brazil, Cuba, South Africa and Spain (Caugant, 1998).

\section{Age and sex distribution}

The median age of the patients was 14 years (range 8 weeks to 83 years). Males constituted $51 \%$ of the patients. Of the total notified cases $164(70 \%)$ of the patients were less than 20 years old. These were distributed between age groups, with $15(6 \%)$ younger than 1 year, $62(27 \%)$ aged $1-9$ years and $87(37 \%)$ aged 10-19 years. Overall, the cumulative incidence rate was highest in infants $(<1$ year of age) at 25.4 (95\% CI 15.3-42.1) per 100000 infants. This was followed by the 10-19 and 1-9 year age groups at 11.1 (95\% CI 9.0-13.7) and 10.2 (95\% CI 7.9-13) per 100000 inhabitants, respectively. The highest age-specific attack rate being found in infants is consistent with other findings across Europe (Trotter et al., 2007) and the USA (Rosenstein et al., 1999).

At the beginning of the hyper-endemic period we observed a significant rise in the incidence of cases involving patients aged 10-19 years in the period 1998-1999, compared with 


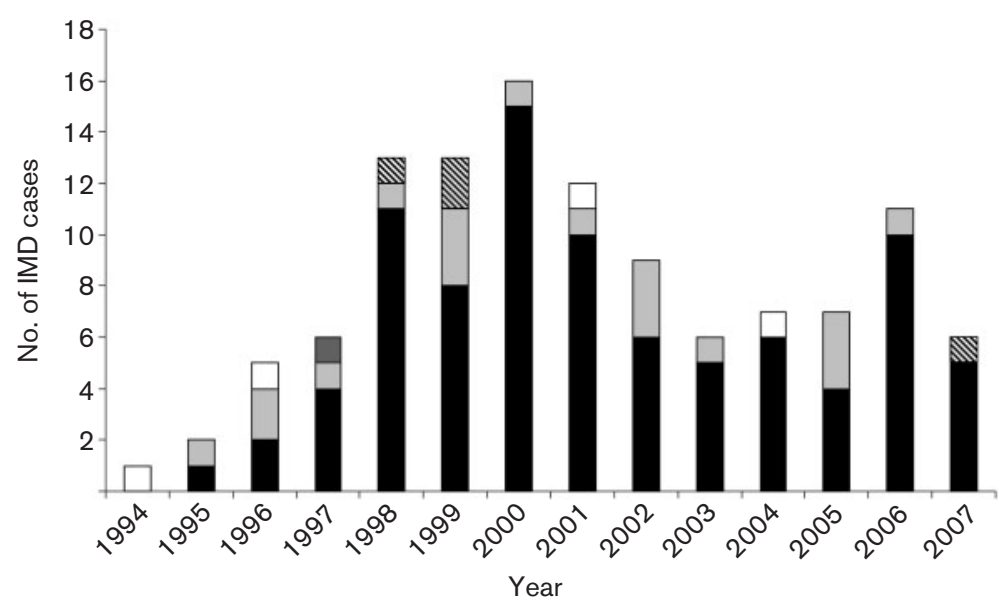

Fig. 2. Annual distribution of IMD cases by serogroup, 1994-2007 ( $n=114)$. Black bars, serogroup $B$; light grey bars, serogroup $C$; hatched bars, serogroup W-135; dark grey bar, serogroup $D$; white bars, non-groupable.

the previous 2 year period $(P<0.005)$ (Fig. 3). A change in the age pattern of patients involving an increased proportion in those over 4 years of age at the start of an epidemic of meningococcal disease has been noted elsewhere (Peltola et al., 1982). Moreover, outbreaks of IMD have been associated with a shift toward disease in school-age children and young adults, and may be caused by exposure, among these age groups, to new strains of $N$. meningitidis to which they were not exposed in earlier childhood (Jackson et al., 1995). Increased transmission may be maintained through factors such as close contact, crowding, exposure to oral secretions and a greater mixing of the people of this population through school and college attendance or leisure activities. Indeed, it has been shown that children and teenagers tend to mix with people of the same age and experience the most intensive close interaction, more than any other age group (Mossong et al., 2008).

\section{Seasonal distribution}

The cumulative number of IMD cases peaked in March $(n=32)$ and again in August $(n=29)$. However, since no significant difference between quarterly periods was found $(P>0.05)$, we could not identify any seasonality of the disease. Malta has a typical Mediterranean climate of mild, wet winters and hot, dry summers. The factors usually associated with winter peaks of IMD in northern European

Table 1. Annual distribution of serogroup B cases by serotype and sero-subtype, 1994-2007 $(n=87)$

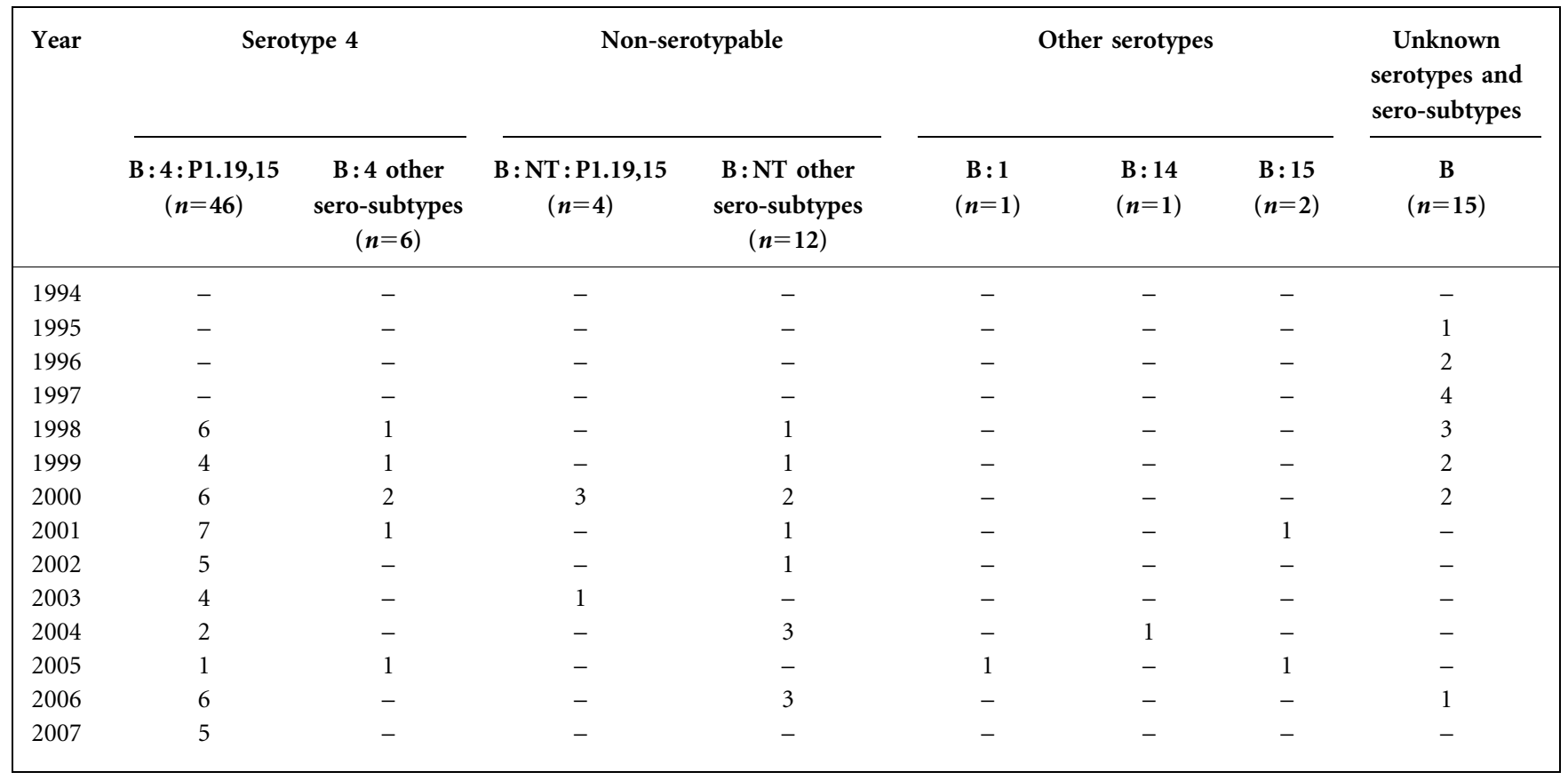

NT, Non-serotypable. 
Table 2. Annual distribution of serogroup C cases by serotype and sero-subtype, 1994-2007 $(n=18)$

\begin{tabular}{|c|c|c|c|c|c|c|c|}
\hline \multirow[t]{2}{*}{ Year } & \multicolumn{2}{|c|}{ Serotype $2 \mathrm{a}$} & \multirow{2}{*}{$\begin{array}{c}\text { Serotype } 2 b \\
\begin{array}{c}\text { C: } 2 b: P 1.5,2 \\
(n=1)\end{array}\end{array}$} & \multicolumn{3}{|c|}{ Non-serotypable } & \multirow{2}{*}{$\begin{array}{c}\begin{array}{c}\text { Unknown } \\
\text { serotypes } \\
\text { and sero- } \\
\text { subtypes }\end{array} \\
\text { C } \\
(n=8)\end{array}$} \\
\hline & $\begin{array}{c}\mathrm{C}: 2 \mathrm{a}: \mathrm{P} 1.5, \mathrm{NT} \\
(n=2)\end{array}$ & $\begin{array}{c}\mathrm{C}: 2 \mathrm{a}: \mathrm{NT}: \mathrm{NT} \\
(n=1)\end{array}$ & & $\begin{array}{c}\mathrm{C}: \mathrm{NT}: \mathrm{NT}, \mathrm{P} 1.10 \\
(n=3)\end{array}$ & $\begin{array}{c}\mathrm{C}: \mathrm{NT}: \mathrm{NT}, \mathrm{P} 1.14 \\
(n=2)\end{array}$ & $\begin{array}{c}\mathrm{C}: \mathrm{NT}: \mathrm{NT}, \mathrm{NT} \\
(n=1)\end{array}$ & \\
\hline 1994 & - & - & - & - & - & - & - \\
\hline 1995 & - & - & - & - & - & - & 1 \\
\hline 1996 & - & - & - & - & - & - & 2 \\
\hline 1997 & - & - & - & - & - & - & 1 \\
\hline 1998 & - & - & - & - & - & - & 1 \\
\hline 1999 & - & 1 & - & - & - & - & 2 \\
\hline 2000 & - & - & 1 & - & - & - & - \\
\hline 2001 & - & - & - & 1 & - & - & - \\
\hline 2002 & - & - & - & 1 & - & 1 & 1 \\
\hline 2003 & 1 & - & - & - & - & - & - \\
\hline 2004 & - & - & - & - & - & - & - \\
\hline 2005 & - & - & - & 1 & 2 & - & - \\
\hline 2006 & 1 & - & - & - & - & - & - \\
\hline 2007 & - & - & - & - & - & - & - \\
\hline
\end{tabular}

NT, Non-serotypable.

countries such as influenza A (Cartwright et al., 1991) and household crowding (Baker et al., 2000) are possibly the same as those occurring during winter in Malta. The occurrence of IMD outside the winter months may be related to factors such as relative humidity as has been reported in Israel (Block et al., 1993), another Mediterranean country with a more comparable climate.

\section{Disease classification and outcome}

Of all notified cases, 59 (25\%) were classified as meningitis, $84(36 \%)$ as septicaemia; the latter included 1 patient who also suffered pneumonia. Features of both meningitis and septicaemia were present in 90 (39\%) cases. There was no statistical difference in disease classification

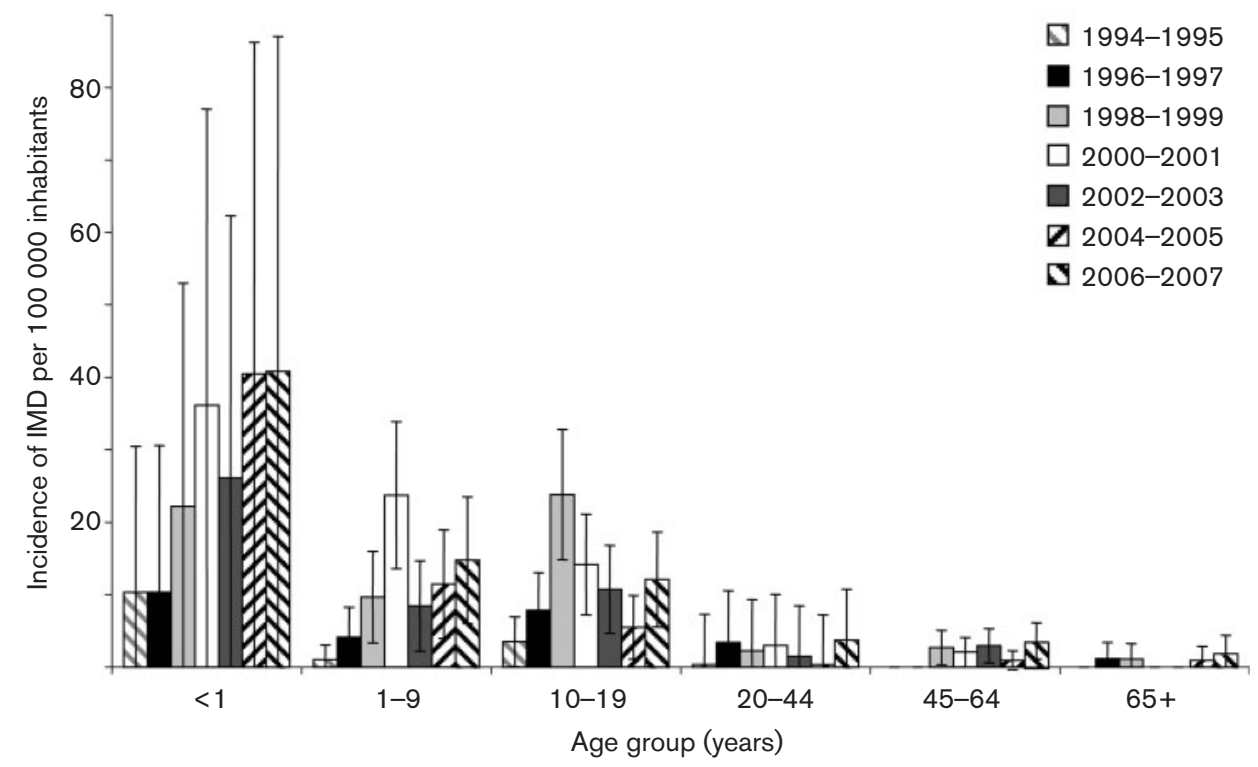

Fig. 3. Incidence of IMD by age group and year group, 1994-2007 ( $n=233)$. The error bars indicate $95 \% \mathrm{Cl}$. 
between the prevailing two serogroups $\mathrm{B}$ and $\mathrm{C}(P>0.05)$. The outcome of the disease was known for all cases. With 28 deaths attributed to IMD the overall CFR was $12 \%$ ( $95 \%$ CI 8-17), which is comparable to the overall CFR of $8 \%$ described in Europe (Trotter et al., 2007). Two of the deaths did not have any laboratory evidence to confirm IMD; however, due to their clinical presentation of septicaemia and rapid progress to death they were attributed to IMD. Although significance testing is precluded owing to the small numbers of cases in the initial years, a downward trend in CFR was observed from the 3 deaths out of 7 IMD cases (43\%) in 1994-1995 to 0 deaths out of the 47 IMD cases in 2006-2007. The CFR was highest in those aged $>45$ years $(23 \%, n=7)$ and lowest in infants $(7 \%, n=1)$. The CFR for those aged $1-9$ years was $6 \%(n=4), 13 \%$ for those aged $10-19$ years $(n=11)$ and $13 \%(n=5)$ for those aged 20-44 years. Other European countries reported an overall higher CFR in the older age groups (EU-IBIS, 2007). Those cases that were classified as septicaemia had the highest CFR $(26 \% ; n=22)$ followed by those with features of both meningitis and septicaemia $(4 \%, n=4)$. The CFR for those classified as meningitis was $3 \%(n=2)$.

Compared with serogroup B, IMD due to serogroup C has been associated with a higher case fatality (Ramsay et al., 1997). However, we found no significant difference in mortality between deaths caused by serogroup B $(n=13)$ and serogroup C $(n=5) \quad(P>0.05)$, possibly because the small numbers studied preclude significance testing.

Apart from stressing the importance of maintaining high vigilance for IMD, our findings underscore the need for enhancing laboratory surveillance of the disease in Malta with the characterization of meningococcal isolates. Almost a third of notified IMD cases did not have a laboratory identification of meningococci to support the diagnosis. The yield of culture-positive isolates may have been adversely affected by antibiotic administration prior to obtaining clinical specimens for culture. Nevertheless, other diagnoses with similar clinical features mimicking early IMD, such as those of viral meningitis, are also possible.

Since spotted fever rickettsiosis is endemic in Malta (Tonna et al., 2006), and can mimic IMD with its clinical presentation of fever, rash and headache, laboratory diagnostics are crucial to ensure that IMD is not missed. Without the isolation of meningococci from blood, the diagnosis of IMD would have been impossible in the two patients originally diagnosed with rickettsiosis. The positive clinical response to ciprofloxacin may have added further support to the mistaken diagnosis of rickettsiosis. Fortunately, ciprofloxacin is also active against meningococci, even though it is not the antibiotic of choice for the treatment of IMD.

The classic methods of laboratory diagnosis based on bacteriological culture remain the mainstay for the correct diagnosis of IMD and characterization of meningococci in Malta. Therefore, clinical specimens from normally sterile sites should be obtained in all suspected cases. The submission of throat swabs and material from haemorrhagic skin lesions is also encouraged as it has been shown that the yield of meningococci is, respectively, about $40 \%$ (Cartwright et al., 1992) and around 63\% (Van Deuren et al., 1993), and is relatively unaffected by any previously administered antibiotics.

Integrating epidemiological data with enhanced laboratory investigations allows for a better understanding of IMD epidemiology. Furthermore, the characterization of meningococci would support the use of immunization to prevent and control the disease, if found to be vaccine-preventable. Several European countries, such as Belgium, Iceland, Ireland, the Netherlands, Spain and the UK, introduced universal and routine conjugated serogroup $C$ vaccination in their respective childhood vaccination programmes (EU-IBIS, 2007). All had higher pre-vaccination incidence rates of serogroup $\mathrm{C}$ meningococcal disease than the annual mean incidence of 0.34 per 100000 inhabitants we observed during the study period.

Implementing the conjugated serogroup $\mathrm{C}$ vaccine in the Maltese childhood vaccination programme at the present level may not be cost-effective. However, if serogroup C meningococcal disease incidence rises or clusters in specific age groups or areas, mass vaccination should be considered. The USA Centers for Disease Control and Prevention (CDC) recommends a threshold for mass vaccination if the primary disease attack rate exceeds $\geqslant 10$ cases per 100000 inhabitants among residents of the same area and who are not close contacts of each other (Bilukha \& Rosenstein, 2005; CDC, 1997). Vaccines against specific meningococcal B strains that were also found in Malta have been licensed for use elsewhere or are under trial (Pace et al., 2008). However, it is the eventual development of a universally safe, immunogenic and effective vaccine against a wide range of meningococcal $\mathrm{B}$ strains that would be a major public-health tool for preventing IMD. Nonetheless, the main methods of controlling the disease remain the early diagnosis and treatment of cases, and the prevention of secondary cases.

\section{ACKNOWLEDGEMENTS}

For their generous contribution towards this study we are indebted to the staff at IDCU, Malta, particularly Anthony Gatt and Peter Grech for assisting in the data collection, Michael Howitz and Caroline Trotter for their helpful comments, and the staff at the Meningococcal Reference Unit, UK, particularly Anthony Carr and Edward Kaczmarski, for further characterization of meningococci. We extend our gratitude to James Stuart for his suggestions early in the drafting of this paper and Kathleen England for obtaining references to historical data.

\section{REFERENCES}

Ashton, F. E., Ryan, J. A., Borczyk, A., Caugant, D. A., Mancino, L. \& Huang, D. (1991). Emergence of a virulent clone of Neisseria 
meningitidis serotype $2 \mathrm{a}$ that is associated with meningococcal group C disease in Canada. J Clin Microbiol 29, 2489-2493.

Baker, M., McNicholas, A., Garrett, N., Jones, N., Stewart, J., Koberstein, V. \& Lennon, D. (2000). Household crowding a major risk factor for epidemic meningococcal disease in Auckland children. Pediatr Infect Dis J 19, 983-990.

Berron, S., De La Fuente, L., Martin, E. \& Vázquez, J. A. (1998). Increasing incidence of meningococcal disease in Spain associated with a new variant of serogroup C. Eur J Clin Microbiol Infect Dis 17, 85-89.

Bilukha, O. O. \& Rosenstein, N. (2005). Prevention and control of meningococcal disease: recommendations of the Advisory Committee on Immunization Practices (ACIP). MMWR Recomm Rep 54, 1-21.

Block, C., Roitman, M., Bogokowsky, B., Meizlin, S. \& Slater, P. E. (1993). Forty years of meningococcal disease in Israel. Clin Infect Dis 17, 126-132.

Cartwright, K. A., Jones, D. M., Smith, A. J., Stuart, J. M., Kaczmarski, E. B. \& Palmer, S. R. (1991). Influenza A and meningococcal disease. Lancet 338, 554-557.

Cartwright, K., Reilly, S., White, D. \& Stuart, J. (1992). Early treatment with parenteral penicillin in meningococcal disease. BMJ 305, 143-147.

Caugant, D. A. (1998). Population genetics and molecular epidemiology of Neisseria meningitidis. APMIS 106, 505-525.

CDC (1997). Control and prevention of serogroup C meningococcal disease: evaluation and management of suspected outbreaks: recommendations of the Advisory Committee on Immunization Practices (ACIP). MMWR Recomm Rep 46, 13-21.

COS (1971-1986). Demographic Review of the Maltese Islands 19701985, annual reports. Central Office of Statistics, Malta.

Connolly, M. \& Noah, N. (1999). Is group C meningococcal disease increasing in Europe? A report of surveillance of meningococcal infection in Europe 1993-6. Epidemiol Infect 122, 41-49.

DH (1933-1970). Annual Reports on the Health Conditions of the Maltese Islands and on the Work of the Medical and Health Department for the Years 1932-1969, annual reports. Department of Health, Malta.

Diggle, M. A. \& Clarke, S. C. (2006). Molecular methods for the detection and characterization of Neisseria meningitidis. Expert Rev Mol Diagn 6, 79-87.

EU-IBIS (2007). Invasive Neisseria meningitidis in Europe 2006. London: Health Protection Agency. European Union Invasive Bacterial Infections Surveillance Network, www.euibis.org (accessed 12 June 2009).

Gray, S. J., Trotter, C. L., Ramsay, M. E., Guiver, M., Fox, A. J., Borrow, R., Mallard, R. H. \& Kaczmarski, E. B. (2006). Epidemiology of meningococcal disease in England and Wales 1993/94 to 2003/04: contribution and experiences of the Meningococcal Reference Unit. J Med Microbiol 55, 887-896.

Jackson, L. A., Schuchat, A., Reeves, M. W. \& Wenger, J. D. (1995). Serogroup $C$ meningococcal outbreaks in the United States: an emerging threat. JAMA 273, 383-389.

Kremastinou, J., Tzanakaki, G., Kansouzidou, A., Pagalis, A., Danielides, V., Kouppari, G., Lada, E., Kriz, P., Musilek, M. \& other authors (1999). Recent emergence of serogroup $C$ meningococcal disease in Greece. FEMS Immunol Med Microbiol 23, 49-55.

Krïzová, P., Musilek, M. \& Kalmusová, J. (1997). Development of epidemiological situation in invasive meningococcal disease in the Czech Republic caused by emerging Neisseria meningitidis clone ET15/37. Cent Eur J Public Health 5, 214-218.

Lind, I. \& Berthelsen, L. (2005). Epidemiology of meningococcal disease in Denmark 1974-1999: contribution of the laboratory surveillance system. Epidemiol Infect 133, 205-215.
Mossong, J., Hens, N., Jit, M., Beutels, P., Auranen, K., Mikolajczyk, R., Massari, M., Salmaso, S., Tomba, G. S. \& other authors (2008). Social contacts and mixing patterns relevant to the spread of infectious diseases. PLoS Med 5, e74. doi:10.1371/journal.pmed.0050074

NSO (1987-1994). Demographic Review 1986-1993, annual reports. National Statistics Office, Malta (formerly the Central Office of Statistics, Malta).

Pace, D., Cuschieri, P., Galea Debono, A. \& Attard-Montalto, S. (2008). Epidemiology of pathogenic Neisseria meningitidis serogroup B serosubtypes in Malta: implications for introducing PorA based vaccines. Vaccine 26, 5952-5956.

Peltola, H., Kataja, J. M. \& Mäkelä, P. H. (1982). Shift in age distribution of meningococcal disease as predictor of an epidemic. Lancet 2, 595-597.

Poolman, J. T., Lind, I., Jónsdóttir, K., Frøholm, L. O., Jones, D. M. \& Zanen, H. C. (1986). Meningococcal serotypes and serogroup B disease in north-west Europe. Lancet 2, 555-558.

Ramsay, M., Kaczmarski, E., Rush, M., Mallard, R., Farrington, P. \& White, J. (1997). Changing patterns of case ascertainment and trends in meningococcal disease in England and Wales. Commun Dis Rep CDR Rev 7, R49-R54.

Rosenstein, N. E., Perkins, B. A., Stephens, D. S., Lefkowitz, L., Cartter, M. L., Danila, R., Cieslak, P., Shutt, K. A., Popovic, T. \& other authors (1999). The changing epidemiology of meningococcal disease in the United States, 1992-1996. J Infect Dis 180, 1894-1901.

Rosenstein, N. E., Perkins, B. A., Stephens, D. S., Popovic, T. \& Hughes, J. M. (2001). Meningococcal disease. N Engl J Med 344, $1378-1388$.

Russell, J. E., Urwin, R., Gray, S. J., Fox, A. J., Feavers, I. M. \& Maiden, M. C. J. (2008). Molecular epidemiology of meningococcal disease in England and Wales 1975-1995, before the introduction of serogroup C conjugate vaccines. Microbiology 154, 1170-1177.

Savona-Ventura, C. (1994). An outbreak of cerebrospinal fever in a 19th century British Mediterranean naval base. J R Army Med Corps 140, 155-158.

Sharip, A., Sorvillo, F., Redelings, M. D., Mascola, L., Wise, M. \& Nguyen, D. M. (2006). Population-based analysis of meningococcal disease mortality in the United States: 1990-2002. Pediatr Infect Dis J 25, 191-194.

Stephens, D. S., Greenwood, B. \& Brandtzaeg, P. (2007). Epidemic meningitis, meningococcaemia, and Neisseria meningitidis. Lancet 369, 2196-2210.

Tikhomirov, E., Santamaria, M. \& Esteves, K. (1997). Meningococcal disease: public health burden and control. World Health Stat Q 50, 170-177.

Tonna, I., Mallia Azzopardi, C., Piscopo, T., Cuschieri, P., Fenollar, F. \& Raoult, D. (2006). Characterisation of rickettsial diseases in a hospital-based population in Malta. J Infect 53, 394-402.

Trotter, C. L., Chandra, M., Cano, R., Larrauri, A., Ramsay, M. E., Brehony, C., Keith, A., Jolley, K. A., Maiden, M. C. J. \& other authors (2007). A surveillance network for meningococcal disease in Europe. FEMS Microbiol Rev 31, 27-36.

Van Deuren, M., Van Dijke, B. J., Koopman, R. J. J., Horrevorts, A. M., Meis, J. F. G. M., Santman, F. W. \& Van der Meer, J. W. (1993). Rapid diagnosis of acute meningococcal infections by needle aspiration or biopsy of skin lesions. BMJ 306, 1229-1232.

Van Deuren, M., Brandtzaeg, P. \& Van der Meer, J. W. (2000). Update of meningococcal disease with emphasis on pathogenesis and clinical management. Clin Microbiol Rev 13, 144-166. 